\title{
Decision Making and Fundamental Matrix approach in Process Safety
}

\author{
Djordje N. Dihovicni* \\ Control Systems, Visoka tehnicka skola, Bulevar Zorana Djindjica 152 a \\ Belgrade, 11070, Serbia ${ }^{\dagger}$ \\ E-mail:ddihovic@Eunet.rs \\ www.visokatehnicka.edu.rs \\ Received 18 October 2010; accepted 23 March 2013
}

\begin{abstract}
The paper describes solving problem of constructing knowledge database of a decision making in process safety. It provides analyses of the requirements as well the analyses of the system incidents caused by specification, design and the implementation of the project. Main focus of this scientific paper is highlighted on practical stability problem and conditions for optimal performance of safe fault-tolerant controllers, $\mathrm{I} / \mathrm{O}$, engineering and pressure transmitters. Algorithm of decision making in process safety is developed and the system has been realized taking into account C\# approach in Windows environment.
\end{abstract}

Keywords: decision making, safety, process, stability. 


\section{Introduction}

One of the most important tasks in the safety engineering lays in the construction of a knowledge database of decision support for the chemical plants, and on that way to ensure optimal conditions, improve quality and boost efficiency. Methods of analysis of control systems and simulation methods, which are used for observing dynamic behavior of linear systems with time delay, and distributed parameter systems, based on linear algebra, operation calculus, functional analyse, integral differential equations and linear matrix nonequations has shown long ago that modern electronic components can be used to achieve more consistent quality at lower cost in safety engineering. The main idea to do so is that the quality service is maintained and controlled. Applying the Fuzzy theory in decision making has given very good results, and provided a flexible framework and over the years numerous mathematical models have been developed.

Almost every chemical plant is well protected, and there always exists plans for securing working places. There is one more aspect of security in process safety- the cyber security. It is extremely important to keep computers and networks free from viruses and hackers, so finding the vulnerabilities of control systems are getting to be a main task in safety and security process.

There are two basic problems to solve in decision making situations: obtaining alternative, and achieving consensus about solution from group of experts. First problem takes into account individual information which existed in collective information units. The later usually means an agreement of all individual opinions. Usually it is considered two approaches for developing a choice process in solving of decision making problems: a direct approach where solution is derived on the basis of the individual relations and as well indirect approach where solution is based on a collective preference relation. In safe engineering technical and economic benefits over hard-wired, discrete components has shown PLC. Main problem in process engineering is practical stability of the system. Chosen system should be stable in required period of time, and this important task is obtained by using practical stability theory for distributed parameter systems. Most systems in chemical engineering as chemical plants for instance, are described by partial different equations and they belong to group of distributed parameter systems.
Taking into account, that the most mathematical models of modern process systems are described by using partial different equations of higher order, and that they belong to distributed parameter systems and as well to time-delay systems, in this paper our first concern was to resolve practical stability from distributed and timedelay perspective. In this scientific paper, it is presented decision support model in process safety. It is developed efficient program support, for complex systems presented by partial different equations of higher order and fundamental matrix approach for practical stability issues. The results obtained by fundamental matrix approach are very near to real values, so the theory is applicable and has wide-spread use in determination where the system stable is. Object oriented approach in Windows environment is applied and showed excellent results. Further investigation will be focusing on development of more complex decision support models and faster program supports, for solving them. Applying adequate decision support models would open the field for discussion how to combine and solve problems with systems described with time delay and as well distributed parameter component.

\section{Practical Stability}

During process of analysing and synthesis of control systems fundamental problem is stability. It is wellknown fact, that we can share stability definitions to Ljapunov and non-Ljapunov concepts that have arisen from various engineering needs. The most often case for consideration of control systems is Ljapunov approach, where the system behavior is considered on infinite interval, which in real cases has only academic importance. From strictly engineering point of view it is very important to know the boundaries where system trajectory comes during there's motion in state space. These practical technical needs are responsible for nonLjapunov definitions, and among them is extremely important behaving on finite time interval- practical stability. Taking into account that system can be stable in classic way lacking appropriate quality of dynamic behavior, and because that, it is not applicable, it is important to take system in consideration in relation with sets of permitted states in phase space which are defined for such a problem. In theory of control systems there are demands for stability on finite time interval that from strictly engineering point of view has 
tremendous importance. The basic difference between Ljapunov and practical stability is set of initial states of system $\left(\mathrm{S}_{\alpha}\right)$, Dihovicni,(3), and set of permitted disturbance $\left(S_{\varepsilon}\right)$ in state space, for every opened set $S_{\beta}$ of permitted states.

Taking into account principle of practical stability, the following conditions must be satisfied:

- determine set $S_{\beta}$ - find the borders for system motion,

- determine set $S_{\varepsilon^{-}}$find maximum amplitudes of possible disturbance,

- determine set $S_{\alpha}$ of all initial state values.

Let us consider $\mathrm{n}$ - dimensional non-linear vector equation:

$$
\frac{d \underline{x}}{d t}=f(\underline{x})
$$

for $d x / d t=0$, solution of this equation is $\underline{x}_{s}=0$ and we can denote it as equilibrium state. Equilibrium state $\underline{\mathrm{x}}_{\mathrm{r}}=0$ is stable in sense of Ljapunov if and only if for every constant and real number $\varepsilon$, exists $\delta(\varepsilon)>0$ and the following equation is fulfilled, Dihovicni (4):

$$
\left\|\underline{x}_{0}\right\|=\| \underline{x}_{t=0} \leq \delta
$$

for every $\mathrm{t} \geq 0$

$$
\|\underline{x}\|<\varepsilon
$$

If following equation exists:

$$
\left\|\underline{x}_{0}\right\| \rightarrow 0 \text { for } t \rightarrow \infty
$$

then system equilibrium state is asymptotic stable.

System equilibrium state is stable, if and only if exists scalar, real function $\mathrm{V}(\underline{\mathrm{x}})$, Ljapunov function, which for $\|\underline{x}\|<r, r=$ const $>0$, has following features:

a) $V(\underline{x})$ is positively defined

b) $d V / d x$ is negatively semi defined for $t \geq 0$

System equilibrium state is asymptotic stable, if and only if exists:

$$
\frac{d V(\underline{x})}{d t} \text { is negatively defined for } \mathrm{t} \geq 0
$$

Derivation of function $\mathrm{V}(\mathrm{x})$, can be expressed:

$$
\frac{d V(x)}{d t}=\nabla_{x}^{T} V(\underline{x}) \frac{d \underline{x}}{d t}=\nabla_{x}^{T} V(\underline{x}) \cdot \underline{f}(\underline{x})
$$

Problem of asymptotic stability of system equilibrium state can be solved for distributed parameter systems, which are described by equation:

$$
\frac{\partial \underline{x}}{\partial}=\underline{f}\left(t, \underline{x}, \frac{\partial \underline{x}}{\partial}, \frac{\partial^{2} \underline{x}}{\partial^{2}} \ldots\right) \quad t \in(0, T)
$$

with following initial conditions:

$$
\underline{x}(t, 0)=\underline{x}_{0}(t)
$$

To satisfy equation (6), space coordinate $z$ cannot be explicitly defined. The solution of equation (6) is $\frac{\partial \underline{x}}{\partial}=\underline{O}$, and let the following equation exists:

$$
\frac{\partial \underline{x}}{\partial}=\underline{x}(t, z)-\underline{x}_{r}(t)
$$

Assumption 1: Space coordinate $\mathrm{z}$ on time interval $\mathrm{t} \in$ $(0, \mathrm{~T})$ is constant.

Accepting previous assumption, and equation (7), we have equation for equilibrium state for system described by equation (6):

$$
\underline{x}_{r}(t)=0
$$

For defining asymptotic stability of equilibrium state the functional $\mathrm{V}$ is used:

$$
V(x)=\int_{0}^{l} V(\underline{x}) d t
$$

where $\mathrm{W}$ is scalar of vector $\underline{\mathrm{x}}$.

We choose functional $\mathrm{V}$ like:

$$
V(x)=\frac{1}{2} \int_{0}^{T} \underline{x} d t
$$

when it is used expression for norm:

$$
\|\underline{x}\|=\sqrt{\int_{0}^{T} \cdot \underline{x} d t}
$$

For asymptotic stability of distributed parameter systems described by equation we use Ljapunov theorems, applied for functional $V$ :

$$
\frac{d V(\underline{x})}{d z}=\int_{0}^{l} T_{x} W(\underline{x}) f t, \underline{x}, \frac{\partial \underline{x}}{\partial}, \frac{\partial^{2} x}{\partial^{2}} \ldots d t
$$




\subsection{Definitions and Conditions of Practical Stability}

Let us consider first order hyperbolic distributed parameter system, which is decribed by the following state- space equation:

$$
\frac{\partial \underline{x}(t, z)}{\partial}=A_{0} \cdot \underline{x}(t, z)+A_{1} \cdot \frac{\partial \underline{x}}{\partial \underline{z}}
$$

with appropriate function of initial state

$$
\begin{aligned}
& \underline{x}_{0}(t, z)=\underline{\psi}_{x}(t, z) \\
& 0 \leq t \leq \tau, O \leq z \leq \zeta
\end{aligned}
$$

where $\underline{x}(t, z)$ is $\mathrm{n}$-component real vector of system state, $\mathrm{A}$ is matrix appropriate dimension, $\mathrm{t}$ is time and $\mathrm{z}$ is space coordinate.

Definition 1: Distributed parameter system described by equation (14) that satisfies initial condition (15) is stable on finite time interval in relation to $[\xi(\mathrm{t}, \mathrm{z}), \beta, \mathrm{T}, \mathrm{Z}]$ if and only if:

$$
\begin{aligned}
& \underline{\psi}_{x}^{T}(t, z) \cdot \underline{\psi}_{x}(t, z)<\xi(t, z) \\
& \forall t \in[0, \tau], \forall z \in[0, \varsigma]
\end{aligned}
$$

then it follows

$$
\begin{aligned}
& \left.\underline{x}^{T}(t, z)\right) \cdot \underline{x}(t, z)<\beta, \\
& \forall t \in[0, T] \forall z \in[0, Z]
\end{aligned}
$$

where $\xi(\mathrm{t}, \mathrm{z})$ is scalar function with feature $0<\xi(t, z) \leq \alpha, 0 \leq t \leq \tau, 0 \leq z \leq \zeta$ where $\alpha$ is real number, $\beta € \mathrm{R}$ and $\beta>\alpha$.

Let calculate the fundamental matrix for this class of system:

$$
\frac{d \Phi(s, \sigma)}{d \sigma}=A_{1} \cdot(s I-A) \cdot \Phi(s, \sigma)
$$

where after double Laplace transformation, and necessary approximation finally it is obtained, Dihovicni, Nedic (5):

$$
\Phi(t, z)=\exp (A \cdot t \cdot z)
$$

where $A=\frac{I-A_{0} \cdot A_{1}}{A_{I}}$.

Theorem1: Distributed parameter system described by equation (1) that satisfies internal condition (2) is stable on finite time interval in relation to $[\xi(\mathrm{t}, \mathrm{z}), \beta, \mathrm{T}, \mathrm{Z}]$ if it is satisfied following condition:

$$
e^{2 \mu(A) \cdot t \cdot z}<\frac{\beta}{\alpha}
$$

Proof: Solution of equation (14) with initial condition (15) is possible to describe as:

$$
\underline{x}(t, z)=\Phi(t, z) \cdot \underline{\psi}(0,0)
$$

By using upper equation it follows:

$$
\begin{array}{r}
\underline{x}^{T}(t, z) \cdot \underline{x}^{T}(t, z)=\underline{\psi}_{x}{ }^{T}(0,0) \cdot \Phi(t, z) \\
\underline{\psi}_{x}{ }^{T}(0,0) \cdot \Phi(t, z)
\end{array}
$$

By using well-known ineqality

$$
\|\Phi(t, z)\|=\|\exp [A \cdot t \cdot z]\| \leq \exp \{\mu(A) \cdot t \cdot z\}
$$

and taking into account that:

$$
\begin{aligned}
& \underline{\psi}_{x}^{T}(0,0) \cdot \underline{\psi}_{x}(0,0)<\alpha \\
& \left\|\underline{\psi}_{x}^{T}(0,0)\right\|=\left\|\underline{\psi}_{x}^{T}(0,0)\right\|<\alpha
\end{aligned}
$$

then it follows:

$$
\underline{x}^{T}(t, z) \cdot \underline{x}(t, z) \leq e^{2 \mu(A t z)} \cdot \alpha
$$

Applying the basic condition of theorem 1 by using equation (19) to further inequality it is obtained, Dihovicni, Nedic (2):

$$
\underline{x}^{T}(t, z) \cdot \underline{x}(t, z)<\frac{\beta}{\alpha} \cdot \alpha<\beta
$$

Theorem 2: Distributed parameter system described by equation (14) that satisfied initial condition (15) is stable on finite time interval in relation to $[\xi(\mathrm{t}, \mathrm{z}), \beta, \mathrm{T}$, $\mathrm{Z}]$ if it is satisfied following condition:

$$
\begin{aligned}
& e^{\mu(A) t} z<\frac{\sqrt{\beta / \alpha}}{1+\tau \cdot \zeta\|A\|} \\
& \forall t \in[0, \tau] \forall z \in[0, \varsigma]
\end{aligned}
$$

The proof of this theorem is given in Dihovicni, Nedic (3).

Let $|\underline{x}|_{(\cdot)}$ is any vector norm and any matrix norm $\|\cdot\|_{2}$ which originated from this vector. Following expresions are used: 


$$
|\underline{x}|_{2}=\left(\underline{x}^{T} \cdot \underline{x}\right)^{1 / 2} \text { and }\|\|_{2}=\lambda_{\max } 1 / 2\left(A^{*} \cdot A\right)
$$

where $*$ and $\mathrm{T}$ are transpose-conjugate and transport matrixes.

It is important to define matrix measure as:

$$
\mu(A)=\lim _{\varepsilon \rightarrow 0} \frac{\|1+\varepsilon \cdot A\|-1}{\varepsilon}
$$

The matrix measure $\mu$ may be defined in three different forms according to the norm which is used:

$$
\begin{aligned}
& \mu_{1}(A)=\max \operatorname{Re}\left(a_{k k}\right)+\sum_{i=1, i \neq k}^{n}\left|a_{i k}\right| \\
& \mu_{2}(A)=\frac{1}{2} \max _{i}\left(A^{T}+A\right) \\
& \mu_{\infty}(A)=\max \operatorname{Re}\left(a_{i i}\right)+\sum_{k=1}^{n}\left|a_{k i}\right|
\end{aligned}
$$

Definition 2: Distributed parameter system described by equation (14) that satisfies initial condition (15) is stable on finite time interval in relation to $[\xi(\mathrm{t}, \mathrm{z}), \beta, \mathrm{T}, \mathrm{Z}]$ if and only if, Dihovicni, Nedic (4):

$$
\left|\underline{\psi}_{x}(t, z)\right|_{2}<\xi(t, z)
$$

then follows

$$
|\underline{x}(t)|_{2}<\beta
$$

where $\xi(\mathrm{t}, \mathrm{z})$ is scalar function with feature $0<\xi(t, z) \leq \alpha, 0 \leq t \leq \tau, 0 \leq z \leq \zeta) \alpha$ is real number, $\beta € \mathrm{R}$ and $\beta>\alpha$.

Theorem 3: Distributed parameter system described by equation (14) that satisfies initial condition (15) is stable on finite time interval in relation to $[\alpha, \beta, T, Z]$ if it is satisfied following condition:

$$
\begin{gathered}
e^{\mu_{2}(A) t z}<\frac{\sqrt{\beta / \alpha}}{1+\mu^{l}{ }_{2}(A)} \\
\forall t \in[0, T] \forall z \in[0, Z]
\end{gathered}
$$

Proof: Solution of equation (14) with initial condition (15) is possible to describe by using fundamental matrix as:

$$
\underline{x}(t, z)=\Phi(t, z) \cdot \underline{\psi}_{x}(0,0)
$$

By using the norms of left and right side of the equation (33) it follows:

$$
\underline{x}^{T}(t, z) \cdot \underline{x}(t, z) \leq e^{2 \mu(A \cdot \bullet \cdot z)} \cdot \alpha
$$

and by using well-known inequality

$$
\begin{gathered}
\|\exp (A \cdot t \cdot z)\|_{2} \leq \exp \{\mu(A \cdot t \cdot z)\} \\
t \geq 0, \quad z \geq 0
\end{gathered}
$$

it follows:

$$
|\underline{x}(t, z)|_{2} \leq e^{\mu_{2}(A) t z} \cdot\left|\underline{\psi}_{x}(O, O)\right|_{2}
$$

and by using equation (30) it is obtained:

$$
|\underline{x}(t, z)|_{2} \leq \alpha \cdot e^{\mu_{2}(A) t z}
$$

so finally it is obtained:

$$
|\underline{x}(t, z)|_{2} \leq \alpha \cdot e^{\mu_{2}(A) t z}\left\{1+\mu_{2}{ }^{l}(A)\right\}
$$

Applying the basic condition of theorem 3 by using equation (32) it is obtained:

$$
\begin{gathered}
|\underline{x}(t)|_{2}<\beta \\
\forall t \in[O, T], \forall z \in[O, Z]
\end{gathered}
$$

Theorem 4: Distributed parameter system described by equation (14) that satisfies initial condition (15) is stable on finite time interval in relation to $[\alpha, \beta, T, Z]$, if it is satisfied following condition, Dihovicni, Nedic (4).

$$
\begin{aligned}
& e^{\mu(A t z)}<\frac{\beta}{\alpha} \\
& \forall t \in[0, T], \forall z \in[0, Z]
\end{aligned}
$$

Theorem 5: Distributed parameter system described by equation (14) that satisfies initial condition (15) is stable on finite time interval in relation to $\left[\mathrm{t}_{0}, \mathrm{~J}, \alpha, \beta, \mathrm{Z}\right]$, if it is satisfied following condition:

$$
\left[1+\left(t-t_{0}\right) \cdot \sigma_{\max }\right]^{2} \cdot e^{2\left(t t_{0}\right)} z \sigma_{\max }<\frac{\beta}{\alpha},
$$

$$
\forall t \in[O, J] \forall z \in[O, Z]
$$

where $\sigma_{\max }$ represents maximum singular value of matrix. The proof of this theorem is given in Dihovicni, Nedic (5). 
In process industry there are also a lot of systems with the time delay, so it is necessary to further investigate this type of systems.

\section{Experiment and results}

The problem of controlling the temperature in a stirred mixing tank is given by experiment, Ray (9). A feedback controller adjusts the fraction of hot stream $\chi(\mathrm{t})$ which is fed to the tank, and it regulates the temperature. The total inlet flow is kept constant by a flow regulator, and the tank level is controlled by an overflow weir. Taking into account a fact that according to the design issues, the hot and cold streams are mixed some distance from mixing tank, there is a transport delay in the inlet feed line.

Control of the temperature in a stirred mixing tank with transport delays in the inlet piping, is shown down below.

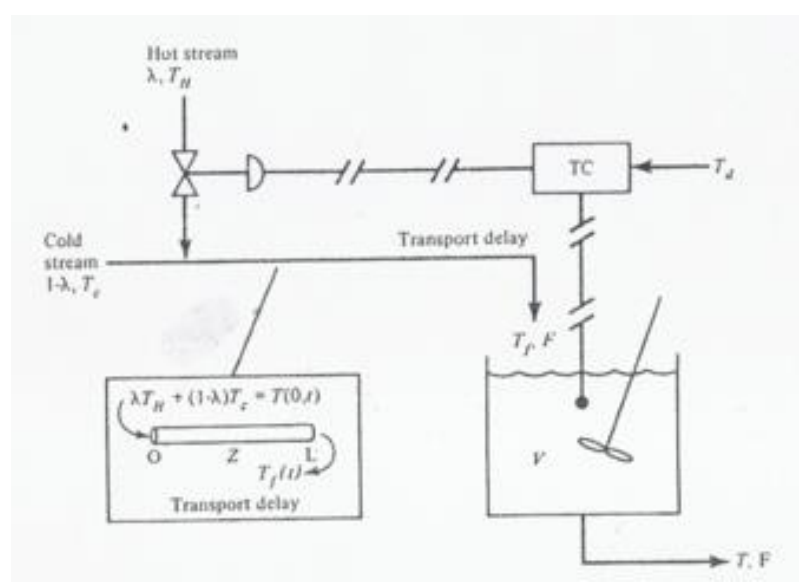

Figure 1. Control of the temperature in a stirred mixing tank with transport delays in the inlet piping

The feed temperature at the mixing tank itself is denoted as $\mathrm{T}_{\mathrm{f}}(\mathrm{t})$, and it is important to calculate temperature $T_{f}(t)$, in terms of the cold-steam temperature $T_{C}$, hot stream temperature $\mathrm{T}_{\mathrm{H}}$, and fraction of hot-stream feed $\chi(\mathrm{t})$. The transport delay occurs due to fluid flowing at flow rate $\mathrm{F}$ in pipe of length $\mathrm{L}$, and cross-sectional area $a_{\mathrm{c}}$.

Mathematical model is presented with hyperbolic equation, and in accordance with experimental results , Ray (9), state space of the distributed parameter system is shown as:

$$
\frac{\partial \underline{x}(t, z)}{\partial t}=\left[\begin{array}{cc}
2 & 1 \\
-2 & 4
\end{array}\right] \frac{\partial \underline{x}}{\partial z}+\left[\begin{array}{cc}
1 & 0 \\
2 & -1
\end{array}\right] \underline{x}(t, z)
$$

where

$$
A_{0}=\left[\begin{array}{cc}
1 & 0 \\
2 & -1
\end{array}\right], A_{1}=\left[\begin{array}{cc}
2 & 1 \\
-2 & 4
\end{array}\right]
$$

and according with equation (28) it is obtained:

$$
A=\left[\begin{array}{cc}
0.548 & 0.904 \\
0.1653 & 3.320
\end{array}\right]
$$

with initial condition:

$$
\underline{x}_{0}(t, z)=\underline{\psi}_{x}(t)=\left[\begin{array}{ll}
0 & 1
\end{array}\right]^{T}
$$

Let us assume following values:

$$
\alpha=2.0 ; \beta=60.22 ; \kappa_{\text {est }}=0.7 \mathrm{~s}
$$

where $\kappa=t \cdot z$, and check initial condition:

$$
\left\|\underline{\psi}_{x}\right\|^{2}=1<2.01
$$

Let us calculate matrix measure $\mu$ and the norm:

$$
\begin{aligned}
& \mu(A)=3.37 \\
& \|A\|=3.45
\end{aligned}
$$

Combining this values into equation (20), it is obtained:

$$
\begin{aligned}
& e^{2 \cdot t \cdot z}<29.96 \\
& \forall t \in[0, T], z \in[0,1]
\end{aligned}
$$

with solution:

$$
\kappa_{e s t}=1.69 s
$$

Upon the theorem 5 it is obtained inequality:

$$
\begin{aligned}
& e^{t \cdot s}<1.23 \\
& \forall t \in[0, T], z \in[0,1]
\end{aligned}
$$

with solution:

$$
\kappa_{e s t}=0.207 \mathrm{~s}
$$

By using theorem 4 it follows:

$$
e^{t \cdot s}<23.31
$$

which solution is:

$$
\kappa_{\text {est }}=3.14 \mathrm{~s}
$$

Upon the theorem 7 it is obtained inequality:

$$
e^{t s}<29.96
$$

and solution is: 
Djordje N. Dihovicni

$$
\kappa_{e s t}=3.39 s
$$

If we use theorem 5 then it is obtained:

$$
[1+3.45 t]^{2} \cdot e^{23.45 t z}<29.96
$$

with solution:

$$
\kappa_{e s t}=0.06 \mathrm{~s}
$$

By analysing the values for estimation, it is obvious that the best result is obtained by using the theorem 5 .

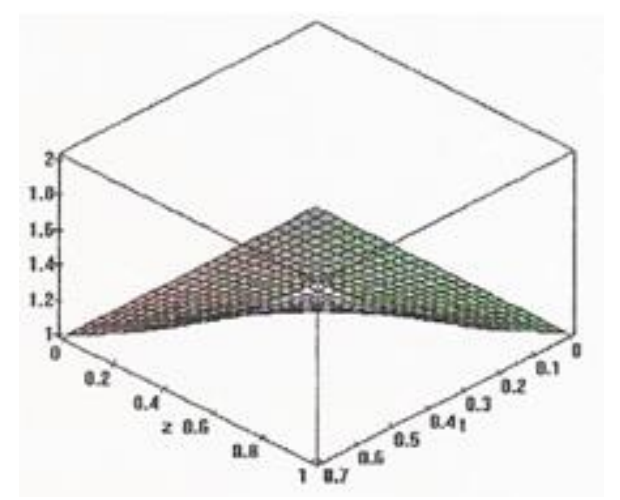

Figure 2. System motion on time interval and space interval z $€[0,1]$

\section{Stability of time delay systems}

Consider the time delay system described by:

$$
\underline{\dot{x}}=A_{0} \cdot \underline{x}(t)+A_{1} \cdot \underline{x}(t-\tau)
$$

with appropriate function of initial state:

$$
x(t)=\underline{\psi}_{x}(t) \quad \tau \leq t \leq 0
$$

where system described by equation (58), is presented in free working state, and $\underline{\mathrm{x}}(\mathrm{t})$ is state vector, and $\mathrm{A}_{0}$ and $A_{1}$ are constants of system matrix of appropriate dimension, and $\tau$ is time delay.

System behaving described by equation (59), is defined on time interval, $J=\left\{t_{0}, t_{0}+T\right\}$, Dihovicni, Nedic (4), and $\mathrm{T}$ can be positive real number, so it may be considered stability and practical stability, and unchangeable sets can be taken as boundary of system trajectories.

Theorem 6: Time delay system (59) with function of initial state (60), is stable on finite time interval in relation with $[\alpha, \beta, \tau, \mathrm{T}]$, if the following conditions are satisfied :

$$
\text { a) }\left\|A_{I}\right\|<\frac{\sigma_{\max }\left(Q^{1 / 2}\right)}{\sigma_{\max }\left(Q^{1 / 2} \cdot P\right)}
$$

where $P$ is solution of matrix equation:

$$
A_{O}^{T} \cdot P+P \cdot A_{O}=2 \cdot Q
$$

and $\sigma_{\max }\left(\mathrm{A}_{0}\right)$ and $\sigma_{\min }\left(\mathrm{A}_{0}\right)$, are maximal and minimal singular values of matrix $\mathrm{A}_{0}$, and holds:

$$
\begin{aligned}
\left\|A_{1}\right\| & <\frac{\sigma_{\max }\left(Q^{1 / 2}\right)}{\sigma_{\max }\left(Q^{1 / 2} \cdot P\right)} \\
\text { b) } e^{\mu\left(A_{0}\right) t} & <\frac{\sqrt{\beta / \alpha}}{1+\tau\left\|A_{l}\right\|}, \forall t \in[O, T]
\end{aligned}
$$

Proof of the conditions a): Consider the functional, presented in Tissir, Hmamed (8):

$$
\begin{aligned}
& v=\underline{x}^{T}(t) \cdot P \cdot \underline{x}(t)+{ }_{t}^{t} f_{\tau}^{T}(s) \cdot Q \cdot \underline{x}(s) \cdot d s \\
& \dot{v}(t)=\underline{x}^{T}(t)\left(A_{0}^{T} \cdot P+P \cdot A_{0}+Q\right) \cdot \underline{x}(t)+2 \cdot \underline{x}^{T}(t) \cdot P \cdot A_{l} \cdot \underline{x}(t-\tau) \\
& \underline{x}^{T}(t-\tau) \cdot Q \cdot \underline{x}(t-\tau) \\
&= \underline{x}^{T}(t) \cdot\left(A_{0}^{T} \cdot P+P \cdot A_{0}+2 \cdot Q\right) \cdot \underline{x}(t)-\underline{x}^{T} \cdot Q \cdot \underline{x}(t)+ \\
&+2 \cdot \underline{x}^{T}(t) \cdot P \cdot A_{l}-\underline{x}(t-\tau)-\underline{x}^{T}(t-\tau) \cdot Q \cdot \underline{x}(t-\tau)
\end{aligned}
$$

From equation (62) and the inequality:

$$
\begin{aligned}
2 \cdot \underline{x}^{T}(t) \cdot P \cdot A_{1} \cdot \underline{x}(t-\tau) & =2 \cdot \underline{x}^{T}(t) \cdot P \cdot A_{1} \cdot Q^{-1 / 2} \cdot Q^{1 / 2} \cdot \underline{x}(t-\tau) \\
& \leq \underline{x}^{T}(t) \cdot P \cdot A_{1} \cdot Q^{-1} \cdot A_{1}{ }^{T} \cdot P \cdot \underline{x}(t)+\underline{x}^{T}(t-\tau) \cdot Q \cdot \underline{x}(t-\tau)
\end{aligned}
$$

it is obtained:

$$
\begin{aligned}
\dot{v} & =-\underline{x}^{T}(t) \cdot Q \cdot \underline{x}(t)+2 \cdot \underline{x}^{T} \cdot P \cdot A_{1} \cdot \underline{x}(t-\tau)-\underline{x}^{T}(t-\tau) \cdot Q \cdot \underline{x}(t-\tau) \\
& \leq-\underline{x}^{T}(t) \cdot Q \cdot \underline{x}(t)+\underline{x}^{T}(t) \cdot P \cdot A_{1} \cdot Q^{-1} \cdot A_{1}^{T} \cdot P \cdot \underline{x}(t) \\
& \leq-\underline{x}^{T}(t) \cdot Q^{1 / 2} \cdot\left(I-Q^{-1 / 2} \cdot P \cdot A_{1} \cdot Q^{-1} \cdot A_{1}^{T} \cdot P \cdot Q^{-1 / 2}\right) \cdot Q^{1 / 2} \cdot \underline{x}(t)
\end{aligned}
$$

where $\dot{v}(t)$ is negative definite if holds equation:

$$
1-\lambda_{\max }\left(Q^{1 / 2} \cdot P \cdot A_{l} \cdot Q^{l} \cdot A_{l}^{T} \cdot P \cdot Q^{1 / 2}\right)>0
$$

which is satisfied if holds following equation:

$$
1-\sigma_{\max }^{2}\left(Q^{1 / 2} \cdot P \cdot A_{1} \cdot Q^{1 / 2}\right)>0
$$

Using the properties, condition (40) holds if:

$$
1-\sigma_{\max }^{2}\left(Q^{1 / 2} \cdot P\right) \cdot \sigma_{\max }^{2}\left(A_{l} \cdot Q^{1 / 2}\right)>0
$$

which is satisfied if: 


$$
1-\frac{\left\|A_{1}\right\|^{2} \cdot \sigma_{\text {max }}^{2}\left(Q^{1 / 2} \cdot P\right)}{\sigma_{\text {min }}^{2}\left(Q^{1 / 2}\right)}>0
$$

and the proof is complete.

By using similar approach:

$$
\left|\int_{a}^{b} \varphi(x) d x\right| \leq \int_{a}^{b}|\varphi(x)| \cdot d x
$$

it is obtained:

$$
\begin{aligned}
\underline{a}^{T}(t) \cdot \underline{c}(t) & \leq\left|\underline{\psi}_{x}^{T}(0) \cdot \Phi^{T} \cdot \int_{-\tau}^{0} \underline{b}(t, \theta) \cdot d \theta\right| \leq\left\|\underline{\psi}_{x}^{T}(0) \cdot \Phi^{T}(t)\right\| \cdot \mid\left\|\int_{-\tau}^{0} \underline{b}(t, \theta) \cdot d \theta\right\| \\
& \leq\left\|\underline{\psi}_{x}^{T}(0)\right\| \cdot\left\|\Phi^{T}(t)\right\| \cdot \int_{-\tau}^{0}\|\underline{b}(t, \theta)\| \cdot d \theta
\end{aligned}
$$

and equation (74) can be written as

$$
\begin{array}{r}
\underline{x}^{T}(t) \cdot \underline{x}(t)=\underline{\psi}_{-x}^{T}(0) \cdot \Phi^{T}(t) \cdot \Phi(t) \cdot \underline{\psi}_{x}(0)+2 \cdot\left\|\underline{\psi}_{x}^{T}(0)\right\| \cdot\left\|\Phi^{T}(t)\right\| . \\
\cdot \int_{-\tau}^{0}\|\Phi(t-\theta-\tau)\| A_{1}\|\| \underline{\psi}_{x}(\theta)\|\cdot d \theta+\| \int_{-\tau}^{0} \underline{b}(t, \theta) d \theta \|^{2}
\end{array}
$$

or:

$$
\begin{aligned}
& \underline{x}^{T}(t) \cdot \underline{x}(t)=\underline{\psi}_{-x}^{T}(0) \cdot \Phi^{T}(t) \cdot \Phi(t) \cdot \underline{\psi}_{x}(0)+2 \cdot\left\|\underline{\psi}_{x}^{T}(0)\right\| \cdot\left\|\Phi^{T}(t)\right\| \cdot \\
& \cdot \int_{-\tau}^{0}\|\Phi(t-\theta-\tau)\| A_{1}\|\|_{-x}(\theta) \| \cdot d \theta+\left[\int_{-\tau}^{0}\|\Phi(t-\theta-\tau)\| A_{1}\|\|_{-x}(\theta) \| \cdot d \theta\right]^{2(76)}
\end{aligned}
$$

If we use well known relation:

$$
m(t) \leq\|\Phi(t-\theta-\tau)\| \cdot\left\|\underline{\psi}_{x}(\theta)\right\| \leq M(t), \forall \theta \in[\tau, 0]
$$

then it yields:

$$
m(t) \bullet \tau \leq\|\Phi(t-\theta-\tau)\| \cdot\left\|\underline{\psi}_{x}(\theta)\right\| \leq M(t) \cdot \tau
$$

It is possible to show that it yields:

$$
\|\Phi(t-\theta-\tau)\|_{\theta \notin \tau, 0]} \leq\|\Phi(t)\| \cdot \cdot\left\|\underline{\psi}_{-x}(\theta)\right\|_{\theta \notin \tau, 0]}<\sqrt{\alpha}
$$

and:

$$
\left\|\underline{\psi}_{x}(o)\right\|<\sqrt{\alpha},\left\|\Phi^{T}(t)\right\|=\|\Phi(t)\|
$$

so the equation (77) can be shown as:

$$
\begin{aligned}
\underline{x}^{T}(t) \cdot x(t) \leq & \leq \Phi(t)\left\|\underline{H}_{x}^{T}(0) \underline{\psi}_{-x}(0)+2 \cdot\right\| \underline{\psi}_{x}^{T}(0)\|\cdot\| \Phi^{T}(t)\|\cdot\| A_{1} \| \cdot \tau \cdot \sqrt{\alpha} \\
& +\left\|\Phi^{T}(t)\right\|^{2} \cdot\left\|A_{1}\right\|^{2} \cdot \tau^{2} \cdot \alpha
\end{aligned}
$$

and if we use well-known inequality, it is given:

$$
\|\Phi(t)\|=\| \exp \left[A_{0}(t) \| \leq \exp \left\{\mu\left(A_{0}\right) \cdot t\right\}\right.
$$

where $\mu$ is defined as:

$$
\mu\left(A_{0}\right)=\frac{1}{2} \cdot \lambda_{\max }\left(A^{T}+A\right)
$$

and if we use the following inequality:

$$
\underline{\psi}_{x}^{T}(0) \cdot \underline{\psi}_{x}(0)<\alpha \cdot\left\|\underline{\psi}_{x}^{T}(0)\right\|=\left\|\underline{\psi}_{x}(0)\right\|<\sqrt{\alpha}
$$

then it yields:

$$
\begin{aligned}
\underline{x}^{T}(t) \cdot \underline{x}(t) & \leq e^{2 \mu\left(A_{0}\right) t} \cdot \alpha+2 \cdot e^{2 \mu\left(A_{0}\right) t}\left\|A_{l}\right\| \cdot \tau \cdot \alpha \\
& +e^{2 \mu\left(A_{0}\right) t}\left\|A_{l}\right\|^{2} \cdot \tau^{2} \cdot \alpha=e^{2 \mu}\left(A_{0}\right) t \cdot \alpha\left(I+\tau\left\|A_{l}\right\|^{2}\right.
\end{aligned}
$$

If we apply theorem basic condition, given by equation (52), to previous inequality, then it is obtained:

$$
\underline{x}^{T}(t) \cdot \underline{x}(t)<\frac{\sqrt{\beta / \alpha}}{1+\tau \cdot\left\|A_{I}\right\|} \cdot \alpha\left(I+\tau \cdot\left\|A_{I}\right\|\right)^{2}<\beta
$$

and thus the proof is complete.

Theorem 7: Time delay system (59) with function of initial state (60), is stable on finite time interval in relation with $\left[\alpha, \beta, \tau, T, \mu_{2}\left(\mathrm{~A}_{0}\right) \neq 0\right]$, if the following conditions are satisfied:

$$
\text { a) } \operatorname{Re}\left[\lambda_{i}\left(A+A_{1} \cdot e^{s \cdot \tau}\right)\right]<0 \quad i=1, \ldots, n
$$

$$
\text { b) } e^{\mu_{2}\left(A_{0}\right) t}<\frac{\beta / \alpha}{1+\mu_{2}{ }^{l}\left(A_{0}\right) \cdot\left\|A_{l}\right\|_{2} \cdot\left(I-e^{\mu_{2}\left(A_{0}\right) \tau}\right)}, \forall t \in[0, T]
$$

In Tissir, Hmamed (8), is given proof of condition $a$.

Theorem 8: Time delay system (59) with function of initial state (60), is stable on finite time interval in relation with $[\alpha, \beta, \tau, T]$, if condition a) of theorem 2 is satisfied, and if it is satisfied the following condition:

$$
1+t \cdot\left\|A_{l}\right\|_{2}<\beta / \alpha, \quad \forall t \in[0, \tau]
$$

Proof: It yields from theorem 2, when $\mu_{2}\left(\mathrm{~A}_{0}\right)=0$.

Proofs for theorem 7, condition $b$ and theorem 8 are given in Dihovicni, Nedic (3). 


\section{Architecture of process safety systems}

There are few well known stages in developing computer decision support systems based on knowledge which include choosing suitable mathematical tools, formalization of the subject area, and development of the corresponding software, Bergmans et etc. (1). In the first phase the problem lays in making right diagnosis and in analyses of the requirements and as well the analyses of the system incidents caused by specification, design and the implementation of the project. The problem of diagnostics may be stated such as finite number of subsets, or it should be applied classical investigation methods. System architecture consists of the following modules:

- cyber security module. Efficient program for investigating viruses and cyber threats,

- $\quad$ stability checking module. This module is designed as program for checking the practical stability of the system, and it consists of two sub-modules for distributed parameter systems and time delay systems. If the system passes this check it goes further to other modules,

- analysis module of safe fault-tolerant controllers, $\mathrm{I} / \mathrm{O}$, engineering and pressure transmitters,

- diagnostics module,

- knowledge module of all possible situations and impacts to chemical plants,

- optimal solution- decision making module,

- presentation module.

For system realization an object oriented programming approach has been used, and the program has been developed using the $\mathrm{C \#}$ language. Each module has a supportive library, Herera et etc (6), and the logical structure is based on the classes, which are described down below for ilustrating purpose.

Main classes are:

- Analyses group which has a primary task of collecting necessary facts about system,

- Cyber security group is used for checking cyber threats,

- Practical stability group which determines if the system is stable or not. If the system is unstable in view of practical stability, then it is automaticly rejected,

- Diagnosis group describes all possible casualities for not required results, or potencial casualities for not optimal costs,

- Performance group is used for the optimal performance.

- Cost group is used for the optimal cost effect.
- Decision making algorithm for optimal performance and cost consists of two phases:

- Phase 1 is used for input Analyses class, Practical stability class and diagnosis class.

- Phase 2 is used for output Performance and Cost group.

The knowledge application is connected to an IBM DB2 Server,where is created a database. The application stores some of its data in traditional database columns, and some of it in native XML columns.

The DB2 database consists of following tables: analyses, practical_stability, diagnosis, performance, cost. The tables also have meta columns and foreign keys, so it is easy to pull data from several tables. The id column in each table is used as the unique identifier for the data in that table. The data column has a type of XML data and holds the main data in XML format.

The procedure of getting XML document from model is presented in the code shown down below..

Creating an $\mathrm{xml}$ document is presented down below.

//Create an xml document

doc $=$ new XmlDocument ()

if (!System.IO.File.Exists(PATH))

\{

//Create necessary nodes

XmlDeclaration declaration $=$

doc.CreateXmlDeclaration("1.0", "UTF-8", "yes");

XmlElement root $=$ doc.CreateElement("Analyses");

XmlElement pipes = doc.CreateElement("Stability");

XmlAttribute diagnosis $=$

doc.CreateAttribute("diagnosis");

XmlElement performance $=$

doc.CreateElement("Performance");

XmlElement cost $=$ doc. CreateElement("Cost");

//Construct the document

doc.AppendChild(root);

root.AppendChild(analyses);

pipes.Attributes.Append(stability);

pipes.AppendChild(diagnosis);

pipes.AppendChild(performance);

doc.Save(PATH);

\}

else

\{

//Create necessary nodes

Doc.LOAD(Path);

XmlElement root $=$ doc. CreateElement $(" S y s t e m ")$;

XmlElement system = doc. CreateElement("Stability"); 


\author{
XmlAttribute analyses $=$ \\ doc.CreateAttribute("analyses"); \\ XmlElement performance $=$ \\ doc.CreateElement("Performance");

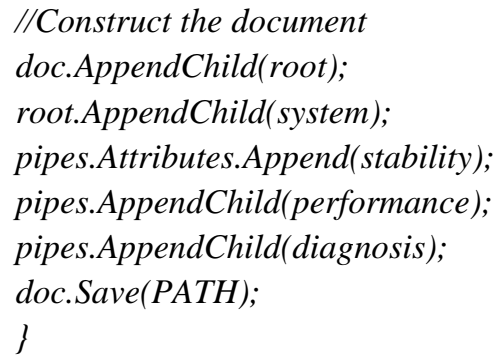

//Construct the document

doc.AppendChild(root);

root.AppendChild(system);

pipes.Attributes.Append(stability);

pipes.AppendChild(performance);

pipes.AppendChild(diagnosis);

doc.Save(PATH);

\}

XmlElement cost $=$ doc. CreateElement $("$ Cost");

\section{Decision support model and cyber security}

The key question of the given problem is in determining how good is a combination of requirements for the function of process safety system, Pérez (10). The first step determines the extent in which the stability is necessary for the performance of the process system.

The second step is focused on analysing the coeficient of satisfaction expressing the extent in which the technology satisfies the requirements. The way in which technologies meet integration requirements is a fuzzy variable, given the adequate description that can be used to quantify the veracity of the hypothesis.

In practice it is very often the case that the conclusions adopted on a diagnostic systems in process safety are wrong, and therefore incorrectly evaluated the working potentials of the system. In order to avoid it, it is mandatory to involve experienced and highly skilled diagnostician in certain situations. However, it can happen that the engagement of experienced and highly trained diagnostician in certain situations is not adequate solution, so the decision models have a crucial role in making diagnostic conclusions about the states of the system in process safety.

The next step is based on monitoring of all possible situations and impacts to process systems in purpose of achieving the optimal performance. The last issue involves cost effect.

These five aspects constitute theoretical basis of the model and they are presented with adequate coefficients $\mathrm{C}_{1}, \mathrm{C}_{2}, \mathrm{C}_{3}, \mathrm{C}_{4}$ and $\mathrm{C}_{5}$. Coefficient $\mathrm{C}$ represents product of the coefficients from $\mathrm{C}_{1}$ to $\mathrm{C}_{5}$.
In this paper it is proposed following semantics for the set of five terms to point different styles of decisions on the strategic management process.

A) $\mathrm{VG}=$ Very Good $=(0.8,0.9,1.0)$

B) $\mathrm{G}=\mathrm{Good}=(0.6,0.7,0.8)$

C) $\mathrm{M}=$ Medium $=(0.4,0.5,06)$

D) $\mathrm{B}=\mathrm{Bad}=(0.2,03,0.4)$

E) $\mathrm{VB}=$ Very $\mathrm{Bad}=(0.0,0.1,0.2)$

The attacks on critical infrastructure can have devastating consequences and are considered to be high value targets for cyber terrorists, so it is necessary to use modern ID responsive to intruders which include log-off an offender or modify firewall setting to block network traffic from malicious source, Walker (11). In case of multistage instrusion attacks in which an intruder would perform multiple attacks at different points, these approaches do not work.

Taking into consideration that most intruders which are making multiple attacks, try to ensure that their action go urecorded, the main thing is generating the sufficient number of alerts. For our cyber security module it is chosen snort, as the most commonly used IDS within cyber infrstructure environment with thousands of alerts per hour. The sensor events are compared against signatures of common similar attacks. The administrators should be aware of the reactive approaches for automatic responses, and the fact that these approaches suffer from a high false alert rate which significantly increaseas their workload, Denning (2).

In the case of cyber infrastructure-oriented environments even the most contemporary IDS are unable to examine encrypted packets or examine large volumes of traffic. The better security could be achieved by incorporation of automatic protection services which are based on the impact to the system and intrusion types.

The snort cyber security model approach resulted in a very limited amount of knowledge within the log file, and examination of all intrusion data including connections that triggered the alarm.

Further improving of current security model is based on host based IDS since they monitor all host activities, and a schema that quantities attacks and provides a domain independent framework which is ideal for quantifyng security threads. 


\section{Conclusion}

By analysing process systems from safety and optimal cost perspective, it is important to recognize which systems are not stable in real conditions. From engineering state of view we are interested in such a systems which are stable in finite periods of time, so our first concern should be to maintain stable and safe systems. Cyber threats in process control systems are very real such as: insufficient network separation between corporate and real-time, security efforts focused solely on corporate internet interface point control systems, remote access to the process control LAN not properly secured, dual-network cards installed and in use, bridging the two networks, null-session authentication, shared folders, and "everyone" permissions defeat any internal IT controls, no outbound filtering of data, workstations / servers running process control applications not properly, process LAN and associated systems easy to find by obvious PC name patched, social engineering and physical security extremely weak, policies either not in place, not followed, inadequate or not enforced and etc, and it is necessary to investigate, find and eliminate it. Our knowledge database is created in DB2, and it involved all possible reasons for non adequate performanse. Key modules for obtaining best performance, safety and the low cost are a good base for the program support in C\# programming language and the UML representation.

\section{References}

1. Bergmans J., Gutnikov S., Krasnoproshin V., Popok S. and Vissia H., Computer-Base Support to Decision-
Making in Orthopaedics, International Conference on Intelligent Technologies in Human related Sciences, vol. 2: Leon, Spain (1996), pp. 217-223.

2. Denning D.E., An Intrusion- Detection Model, IEEETransactions on Software Engineeering, 23(12), pp. 800807.

3. Dihovicni N.Dj., Nedic N., Stability of Distributed Parameter Systems on Finite Space Interval., 32-end Yupiter Conference, Zlatibor (2006), pp. 306-312.

4. Dihovicni N.Dj., Nedic N., Stability of Distributed Parameter Systems on Finite Space Interval described by cubic equations, 32-end Yupiter Conference, Zlatibor (2006), pp. 321-325.

5. Dihovicni N.Dj., Nedic N., Practical Stability of Linear Systems with Delay in State, AMSE, Association for the Advancement of Modelling \& Simulation Techniques in Enterprises, Tassin La-Demi-Lune, France, Vol $62 \mathrm{n}^{0} 2$ (2007), pp. 98-104.

6. Herrera F., Herrera-Viedma E., Lopez E., Computer-Base Support to Decision-Making in Orthopaedics, International Conference on Intelligent Technologies in Human related Sciences, vol. 2, Leon, Spain, (1996), pp. 205-213.

7. Moez, A, A.R., Extreme properties of eigenvalues of hermitian transformation and singular value of the sum and product of linear transformation., (1956), Duke Math. J., 23, pp. 463-467

8. Tissir, E, Hmamed A., Sufficient stability conditions of interval time delay systems, Adv Modelling Anal. (1996), C45, 1-16.

9. Ray H., Advanced process control, McGraw-Hill, (1981).

10. Perez D., Batista J., Decision Support Model for Information Systems Integration in Supply Chains: a Fuzzy Approach, Word Scientific Proceedings Series on Computer Engineering and Information Science, (2010), pp. 281-288.

11. Walker J., Cyber security concerns for emergency management, Intech, (2012), pp.39-60. 